\title{
Schlaganfallprophylaxe
}

\section{Neuer Faktor-Xa-Inhibitor wird nun in Phase III geprüft}

\section{Edoxaban ist ein einmal täglich einzu- nehmender direkter Faktor-Xa-Inhibi- tor, der als potenzielle neue Therapie zur Schlaganfallprävention bei nicht valvulärem Vorhofflimmern und zur Therapie und Prävention rezidivie- render venöser Thromboembolien (VTE) entwickelt wird.}

In der Phase-III- Studie ENGAGE AF-TIMI 48 mit über 21000 Patienten werden derzeit zwei Dosierungen von Edoxaban ( $30 \mathrm{mg}$ und $60 \mathrm{mg}$ ) mit dem Vitamin-K-Antagonisten (VKA) Warfarin verglichen, primärer Endpunkt ist die Nichtunterlegenheit. Es ist die bisher größte Doppelblindstudie mit Antikoagulanzien bei Vorhofflimmern.
Die vor der Einführung der neuen oralen Antikoagulanzien (NOAK) bislang effektivste Therapie mit VKA erfordert ein regelmäßiges INR-Monitoring und kann bei schlechter Einstellung Komplikationen verursachen. So steigt die unter Studienbedingungen angegebene jährliche Rate für schwerwiegende Blutungen von 1,4-3,4\% im Praxisalltag auf bis zu $8 \%$, wobei zerebrale Blutungen im Vordergrund stehen. Daher werde die Entwicklung von NOAK im Allgemeinen begrüßt, sagte Priv.-Doz. Dr. med. Jan Steffel, Kardiologe am Universitätsspital Zürich. Denn sie bieten eine bessere bzw. gleichwertige Effektivität bei größerer Sicherheit, vor allem was Hirnblutungen betrifft. Ein weiterer Vorteil ist die vereinfachte Handhabung durch fixe Dosierung ohne Gerinnungsmonitoring, so Steffel.

Das schlägt sich auch im kürzlich von der European Society of Cardiology (ESC) publizierten fokussierten Update zum Einsatz von NOAK nieder, der zugelassene NOAK aufgrund ihres besseren Nutzen-Risiko-Verhältnisses bei nichtvalvulärem Vorhofflimmern den Vorzug gegenüber VKA gibt. Allerdings sollte aufgrund der teilweise renalen Elimination von NOAK vor Therapiebeginn die Nierenfunktion geprüft werden. (ufo) I

II Symposium, 57. Jahrestagung der Gesellschaft für Thrombose- und Hämostaseforschung (GTH), München, 20. Februar 2013 (Veranstalter: Daiichi Sankyo)

\section{Bioresorbierbares}

\section{Gefäßgerüst}

ABSORB $^{\mathrm{TM}}$ ist das weltweit erste medikamentenfreisetzende bioresobierbare Gefäßgerüst (bioresorbable vascular scaffolds = BVS). Ähnlich wie ein Metallstent sorgt das Implantat dafür, dass das Gefäß geöffnet und der Blutfluss zum Herzen wieder hergestellt wird. Im Gegensatz zu einem dauerhaften Metallstent wird der BVS jedoch innerhalb von etwa zwei Jahren vollständig resorbiert. Wie die kürzlich vorgestellten 3-Jahres-Ergebnisse der ABSORB-Studie zeigen, traten bei Verwendung des BVS im intervenierten Areal während des Beobachtungszeitraums keine Thrombosen auf. Schwerwiegende kardiale Ereignisse (MACE) wurden bei $10 \%$ der Patienten registriert, was der Rate für einen „Best-inclass"-DES entspricht. In einer Patientensubgruppe konnten zudem eine Verbesserung der Vasomotion, eine Lumenerweiterung und eine Plaqueregression festgestellt werden.

(wed)

II Symposium, 79. Jahrestagung der DGK, Mannheim, 4. April 2013 (Veranstalter: Abbott Vascular)

\section{Genauere Elektroden- platzierung bei der CRT}

Trotz aller Fortschritte zeigt sich bei der kardialen Resynchronisationstherapie (CRT) im klinischen Alltag eine mit $30-40 \%$ relativ hohe Nonresponderrate, bemängelte Dr. med. Johannes Sperzel, Kerckhoff-Klinik Herz- und Thoraxzentrum Bad Nauheim. Probleme bei der Implantation und Nachsorge wie hohe Reizschwellen, Phrenikusstimulation (PNS) und Elektrodendislokation seien noch nicht vollends beherrscht. Die neue quadripolare Stimulationstechnologie könne zu einer Verbesserung der CRT-Therapie beitragen und möglicherweise auch die Responderrate erhöhen. Mit bis zu zehn Stimulationsvektoren bietet die vierpolige linksventrikuläre Elektrode (Quartet ${ }^{\mathrm{TM}}$ ) mehr Optionen zur Bestimmung der optimalen Elektrodenposition und zur Behandlung von Stimulationskomplikationen während und nach der Implantation, so Dr. med. Klaus Jürgen Gutleben, Herz- und Diabeteszentrum NRW, Bad Oeynhausen.

(aam) II

II Satellitensymposium , 79. Jahrestagung der DGK, Mannheim, 4. April 2013 (Veranstalter: St. Jude Medical)

\section{Neue Daten zum Klappenersatz}

In der PARTNER-Studie wurde bei 699 Hochrisikopatienten die TAVI mit der Standardoperation am offenen Herzen verglichen. Die minimalinvasive Implantation einer bovinen ballonexpandierbaren Aortenklappe (Edwards SAPIEN) erwies sich nach einem Jahr als nicht unterlegen (Mortalitätsraten: 24,2\% unter TAVI vs. 26,8\% unter Standardoperation; $\mathrm{p}=0,44)$. Auch die 3-Jahres-Daten bestätigen diese Nichtunterlegenheit $(44,2 \%$ vs. $44,8 \% ; p=0,48$ ). Mittlerweile ist die TAVI auch in den Leitlinien zur Behandlung von Herzklappenerkrankungen als Therapieoption anerkannt.

Die Entscheidung über ein jeweils individualisiertes Behandlungskonzept bei einer Aortenklappenstenose sollte stets ein „Herzteam" treffen. Auch ein niedergelassener Kardiologe sollte beteiligt werden. „Die Schlüsselrolle des Niedergelassenen liegt darin, die richtige Diagnose zu stellen", informierte Baumgartner.

(bry) I

II Pressekonferenz, 79. Jahrestagung der Deutsche Gesellschaft für Kardiologie, Mannheim, 4. April 2013 (Veranstalter: Edwards Lifesciences) 